\title{
SOLVING A DISTRIBUTION FACILITY LOCATION PROBLEM USING AN ANALYTIC HIERARCHY PROCESS APPROACH
}

\author{
Jesuk Ko ${ }^{1}$ \\ Department of Industrial and Information Engineering, Gwangju University \\ 592-1 Jinwol-dong, Nam-gu, Gwangju 503-703, Korea \\ jko@gwangju.ac.kr
}

Keywords: facility location, decision factor analysis, analytic hierarchy process

Summary: This paper deals with an integrated decision model for determining the location of distribution facilities. As aids in making distribution location decisions, use of decision factor analysis and the analytic hierarchy process is proposed. The location decision model includes a criterion set having 20 criteria divided in 5 groups. For this study, a questionnaire was developed and given to 180 managers in the distribution arena, asking their opinions about the locations of the distribution facilities. The survey results were analyzed based on the location selection criteria. This application of the location decision model to real-world cases, including the recommendation of new distribution facility locations, demonstrates the practical applicability of the research findings.

\section{Introduction}

Distribution of products to customers is one of the most crucial activities of a manufacturing company. Facilities location and the distribution process are two key components of a distribution system. These two components are interdependent, and it is necessary to consider location and distribution decisions simultaneously. This study proposes the use of decision factor analysis and the analytic hierarchy process (AHP) as aids in making distribution location decisions. The AHP enables the decision maker to structure a complex problem as a simple hierarchy and to evaluate a large number of often conflicting quantitative and qualitative factors in a systematic manner. Our location decision model includes twenty criteria grouped under 5 categories. For this study, a questionnaire was developed and given to 180 managers asking their opinions about the locations of the distribution facilities. The results were analyzed on grounds of the criteria of selecting locations.

As a practical application, several alternatives for the distribution facility locations were evaluated by the location decision model. The goal of this research is to provide decision-makers with a more effective and efficient model for making facility location decisions. In this paper, we show how an integrated decision model can aid location decisions by generating a solution that recognizes the practical considerations while adopting AHP weightings for the decision factors of a qualitative nature. Our integrated decision model is illustrated with a real-world case involving a distribution industry.

The remainder of this paper is structured as follows. In section 2, related literature is reviewed. Section 3 describes a brief overview of the solution methodology, focusing on a wide variety of location decision factors. Based on the AHP and decision modeling approach, the integrated decision model for distribution facility location is presented in section 4. Section 5 addresses the work done to evaluate the existing facilities, and recommends new distribution locations. Finally, in section 6 , some conclusions are included.

\footnotetext{
${ }^{1}$ This study has received partial support from the Industrial Technology Research Center, through the Intramural research fund, at Gwangju University.
} 


\section{Literature Review}

Facility location is one of the popular research topics in decision-making activities. These problems have received much attention over the years and numerous approaches, both qualitative and quantitative, have been suggested. Facility location has a well-developed theoretical background (Baumol and Wolfe, 1958; Brandeau and Chiu, 1989). Generally, research in this area has been focused on optimizing methodology (Brown and Gibson, 1972; Erlenkotter, 1975; Rosenthal, White and Young, 1978; Wesolowsky, 1977). Extensive effort has been devoted to solving location problems employing a wide range of objective criterion and methodology used in the decision analysis. Geoffrion (1978), for instance, includes decomposition, mixed integer linear programming, simulation and heuristics that may be used in analyzing location problems. He notes that a suitable methodology for supporting managerial decisions should be computationally efficient, lead to an optimal solution, and be capable of further testing. Other researchers stress the importance of multiple criteria that must be included in the decision analysis (Erlenkotter, 1975). Many methodologies have been utilized to solve the facility location problem. Baumol and Wolfe (1958) have solved the location problem for minimum total delivery cost with nonlinear programming. Others have incorporated stochastic functions to account for demand and/or supply (Rosenthal, White and Young, 1978; Wesolowsky, 1977). Other approaches that have been employed include dynamic programming (Geoffrion, 1978; Saaty, 1996; Tansel, Francis and Lowe, 1989), multivariate statistics using multidimensional scaling (Asami and Walters, 1989) and heuristic and search procedures (Kuehn and Hamburger, 1963). In many location problems, cost minimization may not be the most important factor. The use of multiple criteria has been thoroughly discussed in the literature (Schniederjans and Garvin, 1997; Sweeny and Tatham, 1976). Researchers have suggested numerous criteria for the facility location problem. These decision factors include availability of transportation facilities, cost of transportation, availability of labor, cost of living, availability and nearness to raw materials, proximity to markets, size of markets, attainment of favorable competitive position, anticipated growth of markets, income and population trends, cost and availability of industrial lands, proximity to other industries, cost and availability of utilities, government attitudes, tax structure, community related factors, environmental considerations, assessment of risk and return on assets. Qualitative factors are crucial but often cumbersome and usually treated as part of management's responsibility in analyzing results rather than quantified and included in a model formulation of the facility location problem (Lee, Green and Kim, 1981). Qualitative decision factors can be readily incorporated into facility location problems if the analytic hierarchical process is employed. Combining decision factor analysis and AHP, this study will analyze the evaluation of location decisions involving distribution facility location factors. Specifically, this research concerns the stage in the decision-making process when the weighted score of potential distribution sites has been ranked to choose an optimal candidate.

\section{The Solution Approach}

In today’s dynamic and volatile global economy, many researchers underline the importance of facility location factors. Issues associated with distribution facility location include political, economic, legal, social and cultural environments. Facility location decisions involve a substantial capital investment and result in long-term constraints on distribution of goods. These problems are complex and, like most realworld problems, depend upon a number of tangible and intangible factors that are unique to the problem. The complexity stems from a multitude of qualitative and quantitative factors influencing location decisions as well as the intrinsic difficulty of making trade-offs among those factors. One analytical approach often suggested for solving such a complex problem is the AHP, first introduced by Saaty (1980, 1988, 1996). It is a highly flexible decision methodology that can be applied in a wide variety of situations (Zahedi, 1996). It is typically used in a decision-making situation involving selecting one or more alternatives from several candidate locations on the basis of multiple decision criteria of a competing or conflicting nature. Particularly important, the decision criteria may hold a different degree of adequacy or level of importance in the eyes of the decision-makers. In this paper, the decision factor analysis is performed first, based on the questionnaires. Then a combined AHP and decision factor model is presented as an extension to evaluate collectively the location criteria in the decision-making process. 


\section{Location Decision Model}

In this section, the integrated decision model for distribution location is presented. Table 1 summarizes the integrated weighted values of the various location decision factors, and the relative rankings among the decision factors.

\section{Table 1. Integrated decision model for distribution facility location}

Criterion Groups $\quad$ Decision Factors
(Ranking)

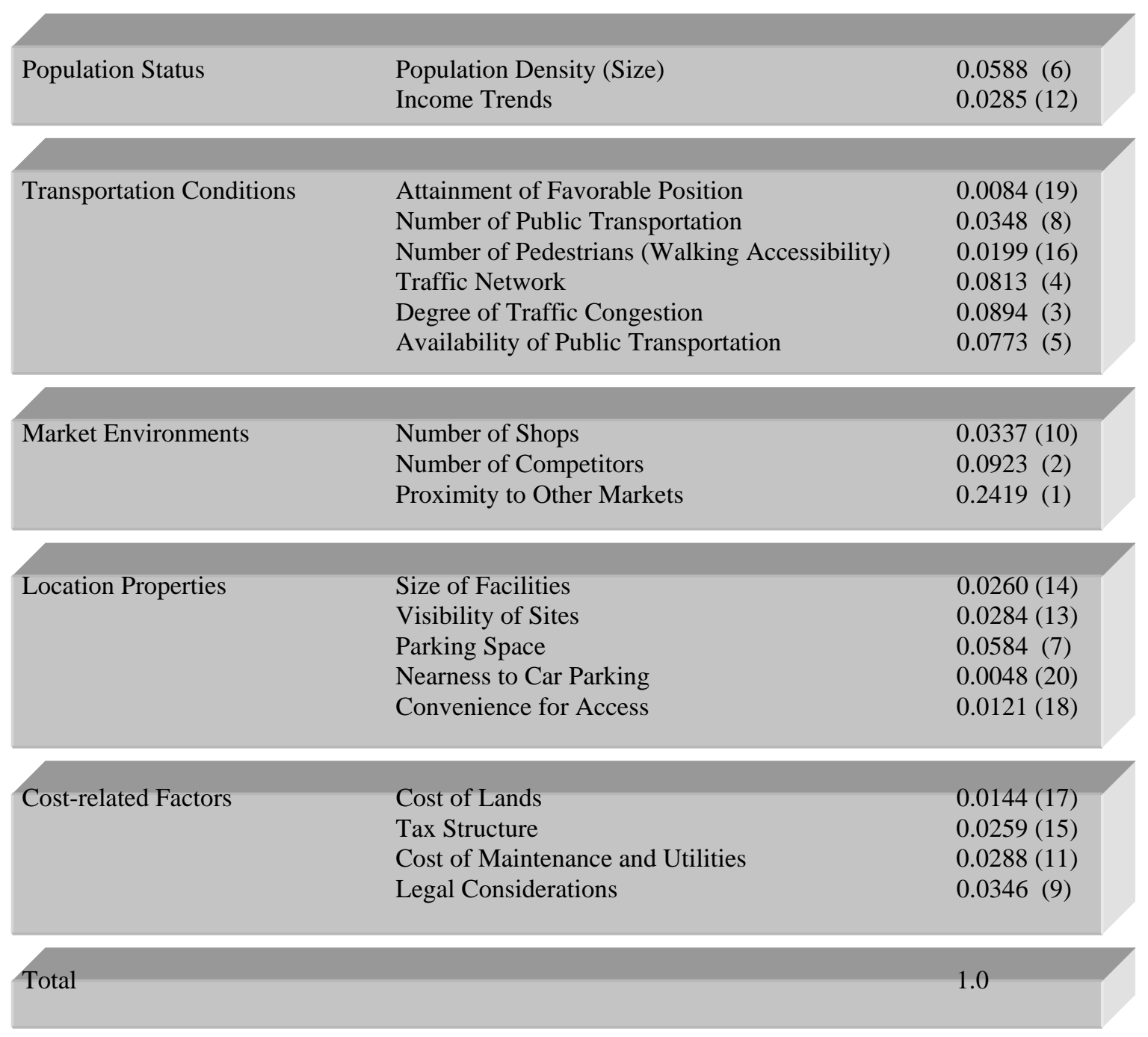

As given in the above table, the competitive market environments such as proximity to other markets and number of competitors are the most crucial factors in the location decision of distribution facilities. Also, traffic congestion, traffic network and availability of public transportation are important decision factors as well. Relatively, cost-related factors and location properties are less influential for deciding a distribution facility location.

\section{A Case Study}


This section addresses the application of the integrated location decision model to realistic examples. Five locations of existing distribution facilities are used for analyzing the adequacy of the established location.

\subsection{Location Evaluation for the Existing Distribution Facilities}

For evaluation, the ways of assigning a weight could be to assign a value ( 0 or 1 ) to each decision factor subjectively, or to do a pairwise comparison $(0,1$ or 2 ) for each pair of decision factors and obtain the weights after applying the integrated location decision model.

\section{Table 2. Overall rating of three distribution locations (subjective assignment)}

Distribution Facilities Integrated Weighting (Total Scores) Evaluation Result

\begin{tabular}{lll} 
Store A & 0.4730 & \\
Store B & $0.8005^{*}$ & Best Location \\
Store C & 0.6326 & \\
\hline
\end{tabular}

From the application of the model, the total score for each existing location by integrated weighting is computed. The results indicate that Store B is considered the best location as provided in Table 2 . Considering the relative comparison of four existing distribution locations, the pairwise comparison in Table 3 shows that Stores D and E exhibit their competitive locations.

\section{Table 3. Overall rating of two distribution locations (pairwise comparison)}

Distribution Facilities Integrated Weighting (Total Scores) Evaluation Result

\begin{tabular}{|lll|} 
Store B & 0.8781 & \\
Store D & $1.1213^{*}$ & Better Location \\
\hline & & \\
\hline Store C & 0.6600 & Better Location \\
\hline Store E & $1.4357^{*}$ & \\
\hline
\end{tabular}

\subsection{Location Decision for New Distribution Facilities}

We consider two locations for new distribution facility alternatives. For pairwise comparisons on the location alternatives, three criterion groups (the population status, the transportation conditions and the market environments) are evaluated.

Table 4. Overall rating of two distribution location alternatives (pairwise comparison)

Location Alternatives Integrated Weighting (Total Scores) Decision Preference

Candidate $\mathrm{X}$

0.7379

Candidate Y

$0.7947^{*}$

Better Choice 
Regarding the recommendation of a potential site, Table 4 reveals that alternative $\mathrm{Y}$ would be a better candidate for the new location of a distribution facility.

\section{Conclusions}

The distribution facility location decision is a more complex problem due to the uncertainty and volatility of distribution environments. The location decision process involves qualitative as well as quantitative factors. Decision makers can no longer ignore the influence of sensitive factors such as the population status of a candidate region, transportation conditions, market surroundings, location properties and cost factors related the alternative location. Moreover, the process could become highly judgmental if a wide variety of qualitative factors are present. In such cases, the selection process may lack consistency and flexibility. The AHP methodology has been employed successfully to provide consistent evaluation (weighting and ranking) of location alternatives. This paper suggested two approaches to the location decision problem: an AHP approach, and an integrated AHP and decision modeling approach. The application presented in this study has illustrated how multiple decision factors can be combined with the AHP approach to permit a more flexible and inclusive use of available information about alternative locations in a facility location decision. The integrated decision model offers a systematic approach to the distribution facility location problem. It extends previous research in the area by incorporating a consistent weighting of crucial factors within an optimization facility location decision process.

\section{References}

Asami, Y. and Walters, I. (1989). Imperfect Information, Uncertainty and Optimal sampling in the Location Theory. Journal of Regional Science. 5, 507-521.

Baumol, W. and Wolfe, P. (1958). A Warehouse Location Problem. Operations Research. 6(2), 252-263.

Brandeau, M. and Chiu, S. (1989). An Overview of Representative Problems in Location Research. Management Science. 35, 645-674.

Brown, P. and Gibson, D. (1972). A Quantified Model for Site Selection-Application to a Multi-plant Location Problem. AIIE Transactions. 4(1), 1-10.

Erlenkotter, D. (1975). Capacity Planning for Large Multi-location System: Approximate and Incomplete Dynamic Programming Approaches. Management Science. 22(3), 274-285.

Geoffrion, A. (1978). A Guide to Computer Assisted Methods for Distribution System Planning. Sloan Management Review. 24(5), 535-544.

Kuehn, A. and Hamburger, M. (1963). A Heuristic Program for Locating Warehouses. Management Science. 9(4), 643-666.

Lee, S., Green, G. and Kim, C. (1981). A Multiple Criteria Model for the Location Problem. Computers and Operations Research. 8(1), 1-8.

Moon, I. and Goldman, A. (1989). Tree Network Location Problems with Minimum Separations. IIE Transactions. 21, 230-240.

Rosenthal, J., White, J. and Young, D. (1978). Stochastic Dynamic Location Analysis. Management Science. 24(6), 645-653.

Saaty, T. (1980). The Analytic Hierarchy Process. New York, McGraw-Hill. 
Saaty, T. (1988). Decision Making for Leaders. Pittsburgh, RWS Publications.

Saaty, T. (1996). Multi-criteria Decision Making: The Analytic Hierarchy Process. Pittsburgh, RWS Publications.

Schniederjans, T. and Garvin, T. (1997). Using the Analytic Hierarchy Process and Multi-Objective Programming for the Selection of Cost Drivers in Activity-Based Costing. European Journal of Operational Research. 100, 72-80.

Sweeny, D. and Tatham, R. (1976). An Improved Long-run Model for Multiple Warehouse Location. Management Science. 22(4), 748-758.

Tansel, B., Francis, R. and Lowe, T. (1989). Location on Networks: A Survey. Management Science. 29, 482-492.

Wesolowsky, G. (1977). Probabilistic Weights in the One-dimensional Facility Location Problem. Management Science. 24(2), 224-229.

Zahedi, F. (1996). The Analytic Hierarchy Process: A Survey of the Methods and Its Applications. Interfaces. 16(4), 96-108. 